\title{
Evaluation of three cases of mycotic keratitis caused by Fusarium solani sensu stricto (FSSC5)
}

\author{
Hazal Boral ${ }^{1}$, Anne van Diepeningen², Elif Erdem³, Meltem Yağmur ${ }^{3}$,
} G. Sybren de Hoog ${ }^{4,5}$, Macit Ilkit ${ }^{*}$, Jacques F. Meis ${ }^{5,6}$, Abdullah M.S. Al-Hatmi ${ }^{4,5,7}$

${ }^{1}$ Division of Mycology, Department of Microbiology, Faculty of Medicine, University of Çukurova, Adana, Turkey ${ }^{2} \mathrm{BU}$ Biointeractions and Plant Health, Wageningen University and Research, Wageningen, The Netherlands ${ }^{3}$ Department of Ophthalmology, Faculty of Medicine, University of Çukurova, Adana, Turkey

${ }^{4}$ Westerdijk Fungal Biodiversity Institute, Royal Dutch Academy of Arts and Sciences, Utrecht, The Netherlands

${ }^{5}$ Centre of Expertise in Mycology Radboud University Medical Centre / Canisius Wilhelmina Hospital, Nijmegen, The Netherlands

${ }^{6}$ Department of Medical Microbiology and Infectious Diseases and ECMM Excellence Center for Medical Mycology, Canisius Wilhelmina Hospital, Nijmegen, The Netherlands

${ }^{7}$ Ministry of Health, Directorate General of Health Services, Ibri, Oman

\section{Objectives}

Owing to a lack of appropriate diagnostic and therapeutic approaches for mycotic keratitis, approximately one million cases of preventable corneal blindness are reported each year. The number of keratitis cases due to infection with Fusarium is increasing significantly worldwide and many cases are not treated adequately and in a timely manner due to frequent misdiagnosis. Species level identification of Fusarium strains that cause keratitis is important, since antifungal susceptibilities may vary between different species of the genus. In the current report, we describe three keratitis cases caused by Fusarium solani sensu stricto (FSSC5) from Turkey and The Netherlands, following ocular trauma.

\section{Material Methods}

Corneal scrapings from the ulcers (Figure 1) of all of the patients were inoculated as multiple ' $C$ ' streaks on blood agar and brain heart infusion agar plates and incubated at $37^{\circ} \mathrm{C}$; Sabouraud glucose agar and potato dextrose agar plates and incubated at $28^{\circ} \mathrm{C}$. Preliminary identification at the genus or species complex level of the isolates was performed by analysis of macro- and micro-morphology on $2 \%$ malt extract agar, incubated at $24^{\circ} \mathrm{C}$. Further identification of the strains was undertaken by partial sequencing of the elongation factor 1 alpha gene. Antifungal susceptibility testing was performed as described in the CLSI document M38-A2 using the following drugs: amphotericin $B$, fluconazole, itraconazole, voriconazole, posaconazole, isavuconazole, micafungin, and anidulafungin.

\section{Results}

Fungal growth was recorded on SGA and PDA plates as woolly, cream-colored aerial mycelia, and colonies with cream-colored reverse. The strains (CBS 138564, CWZ 5051826267, and CWZ 6080556699) were consequently identified as $F$. solani sensu stricto according to results of a BLAST search against the sequences in GenBank. The etiological agent of keratitis exhibited low minimum inhibitory concentrations (MICs) of $1 \mu \mathrm{g} / \mathrm{mL}$ against amphotericin $\mathrm{B}$, and high MICs above the published epidemiological cut off values for voriconazole $(8 \mu \mathrm{g} / \mathrm{mL})$. Patients were successfully treated with topical amphotericin $\mathrm{B}$ and voriconazole and recovered completely (Figure1).

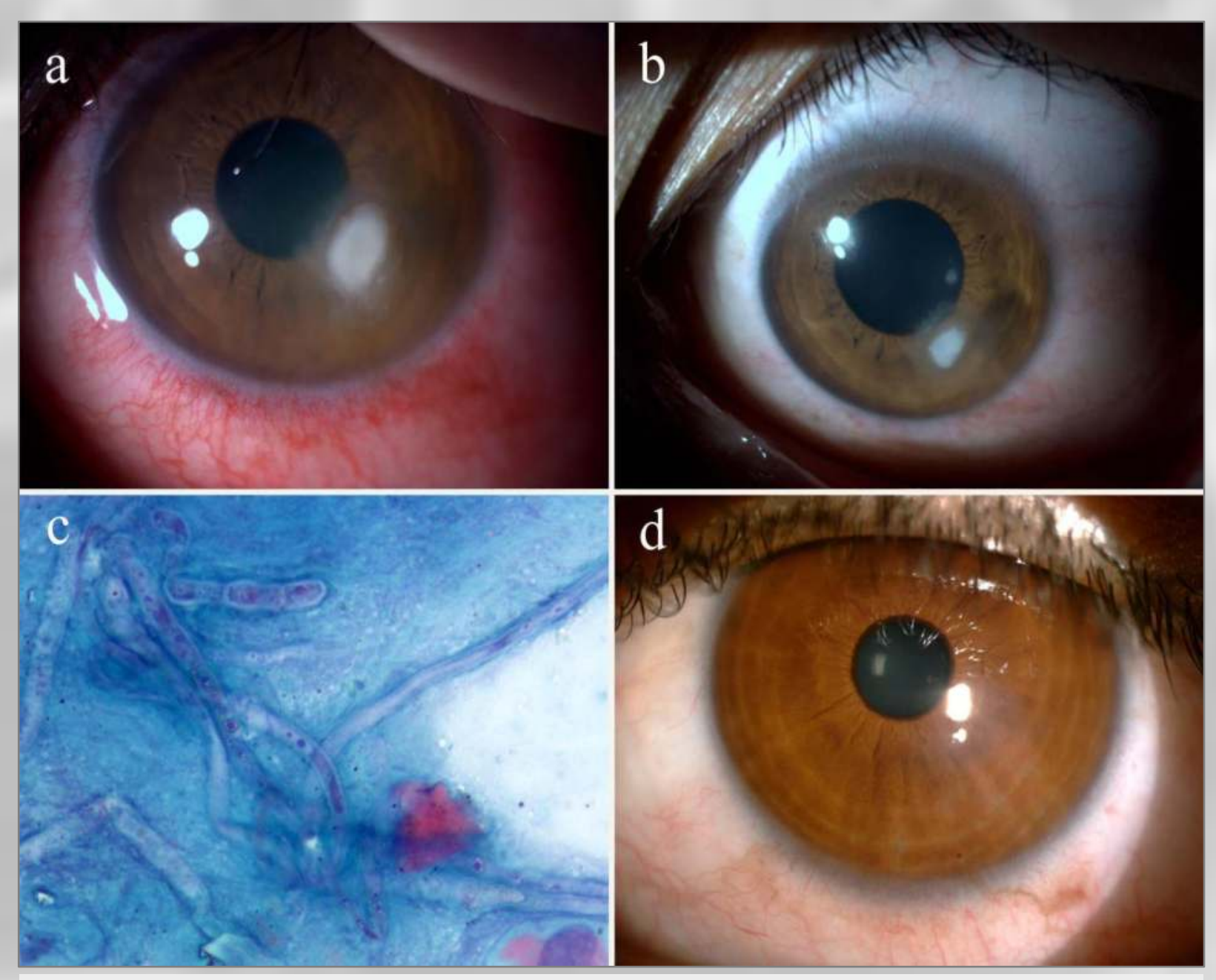

Fig 1. Clinical appearance before $(\mathbf{a}, \mathbf{b})$ and after (d) the treatment and PAS-staining (c) analysis of one of the cases.

\section{Conclusions}

Although $5 \%$ natamycin is currently the most widely used topical polyene for keratitis, especially for Fusarium strains, this drug is not widely used in many countries, including Turkey and The Netherlands. Hence, the combination therapy with voriconazole and natamycin may be an alternative treatment for keratitis caused by Fusarium species. 